\title{
Histological identification of Helicobacter pylori: comparison of staining methods
}

\author{
Pranjal Sharma $\mathbf{R}^{1}$, Kalpana Kumari M.K ${ }^{2}$ \\ ${ }^{1}$ Pranjal Sharma R, MBBS Student, M.S. Ramaiah Medical College, ${ }^{2}$ Dr. Kalpana Kumari MK, Associate Professor, \\ Dept. of pathology, M.S. Ramaiah Medical College, MSR College Road, MSR Nagar, MSRIT Post, Mathikere, \\ Bengaluru, Karnataka 560054, India
}

Address for Correspondence: Dr. Kalpana Kumari MK, Email: kalpank@gmail.com

\begin{abstract}
Introduction: Helicobacter pylori has a high incidence world over and especially in developing countries. It causes many histological derangements apart from causing chronic gastritis. The study was done to compare Haematoxylin $\&$ Eosin (H\&E) and Giemsa with Immunohistochemistry (IHC) for the detection of Helicobacter pylori and to correlate the H. pylori positivity with histological changes. Methods: This study was done in the Department of Pathology on gastric biopsies with clinical suspicion of Helicobacter pylori infection, received in the period between January 2013- December 2013. Sections were stained with H\&E, Giemsa and IHC. Along with detection of the organism associated morphological changes were assessed. The stains were validated using IHC as gold standard. Results: A total of 58 samples were evaluated. Fourteen $(24.1 \%)$ samples showed positivity with IHC, of which $11(19 \%)$ and $9(15.5 \%)$ were positive with H\&E and Giemsa respectively. H\&E had a high false positive rate (19\%). Giemsa staining showed less sensitivity (64.28\%) compared to H\&E (78.57\%). Morphological changes were assessed and organisms were noted in 14(25.5\%) cases with inflammation, $8(30.8 \%)$ cases with activity and $2(28.6 \%)$ cases with atrophy. No organisms were seen associated with intestinal metaplasia. Conclusion: Giemsa was not found to be superior to H\&E in detecting Helicobacter pylori. Use of IHC not only reduces rate of false positive results, but also diagnosis of any mild infection that is not detected on $\mathrm{H} \& \mathrm{E}$ can be made.
\end{abstract}

Keywords: Gastritis, Helicobacter Pylori, Haematoxylin \& Eosin, Special Staining.

\section{Introduction}

Helicobacter pylori is associated with chronic gastritis, ulcers and adenocarcinoma of stomach, [1,2] more so in developing countries. Though $H$. pylori can be detected by invasive and non-invasive tests, [3, 4] histopathological evaluation is the gold standard. [4-6]. Detection in mucosal biopsy is done by staining with $\mathrm{H} \& \mathrm{E}$, but is enhanced by use of histochemical and immunohistochemical stains [1,7]. Many studies have found ancillary stains to be sufficient for diagnosis, [810] but Hartman and Owens [11] contradict this view and suggest the increased use of IHC for $H$. pylori [12, 13].

Therefore, the question of whether ancillary stains

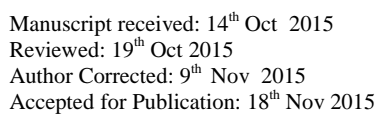

Accepted for Publication: $18^{\text {th }}$ Nov 2015

should be routinely used remains a subject of considerable debate.

\section{Objectives}

1. To compare Hematoxyline and Eosin $(\mathrm{H} \& \mathrm{E})$ and Giemsa with immunohistochemistry for the detection of $H$. pylori.

2. To correlate the $H$. pylori positivity with histological changes.

\section{Materials and Methods}

This study was conducted with a sample size of 58. In the present study, using published data of Tajalli et al., to get a relative precision of $13 \%$ and $95 \%$ confidence level in the result, the study required a minimum of 55 subjects. 
Upper endoscopic biopsies received for dyspepsia and processed in the year 2013 were reviewed. All endoscopic biopsies which have been done for chronic upper abdominal symptoms such as abdominal pain, dyspepsia, heartburn, nausea and vomiting due to suspected $H$. pylori infection were included in the study. Inadequate, autolysed or necrotic tissues and those with extensive areas of haemorrhage were excluded.

Paraffin embedded blocks, of endoscopic biopsy, were retrieved from archives. $3 \mu \mathrm{m}$ thick sections were made and mounted on slides for histochemistry and IHC. Sections were stained with H\&E, Giemsa and IHC by standard procedures. Giemsa stain was used in 1:9 dilutions. IHC was performed using Primary Antibody HPYLORI-L-CE-S $0.1 \mathrm{ml}$ NCL-L-H pylori and Detection Kit RE7290-CE Novolink MinPolymer DS.

The H\&E stained slides were reviewed by two pathologists.

\section{Detection of $\boldsymbol{H}$. pylori and pathology}

1. Patients with positive result in immunohistochemistry were considered to be $H$. pylori positive.

2. Semi-quantitative method of scoring according to the Updated Sydney Classification System [14] was undertaken. The histopathological variables namely, $H$. pylori density and inflammation, were graded as absent, mild, moderate and severe with a scoring of 1 to 4 respectively. Other histopathological features (activity, atrophy and intestinal metaplasia) were not graded, but assessed in case of their presence or absence. H. pylori colonization was assessed and graded after careful search for focal or complete involvement of the gastric surface

3. The biopsy cases were analysed in an attempt to assess the major histopathological features of gastritis. The degree of inflammatory activity was investigated for involvement according to the density of neutrophils in gastric mucosal crypts, from one to all crypts. The presence of mononuclear infiltration was investigated. The degree of intestinal metaplasia was assessed according to the amount of glandular tissue replaced by intestinal type epithelium. Mucosal atrophy was defined as a loss of specialized gastric glands in mucosa, partly replaced by intestinal metaplastic epithelium. It was characterized by architectural changes manifested by variation in the volume and irregularity in the shape, branching, and spacing of the glands.
Statistical Tests done: The statistics of $H$. pylori positivity with $H \& E$ and Giemsa have been analysed and presented in terms of percentage. Validity of H\&E and Giemsa in detection of $H$. pylori was done using sensitivity, specificity, positive and negative predictive values by comparing with IHC as gold standard. Analysis was carried out using SPSS Version 17 software. Inter-observer variation was calculated using Kappa statistic.

\section{Results}

In accordance with the updated Sydney system, the cases showing chronic gastritis were graded into mild $33(56.9 \%)$, moderate $20(34.5 \%)$ and severe inflammation $2(3.4 \%)$. No inflammation was seen in the remaining $3(5.2 \%)$ cases. Of the 58 cases 26 $(44.8 \%)$ cases showed activity, glandular atrophy was noted in $7(12.1 \%)$ cases and intestinal metaplasia in 12 $(20.7 \%)$ cases.

With H\&E (fig.1) H. pylori organisms were detected in $22(38 \%)$ cases. $11(19 \%)$ cases were found to be false positive and $3(5.2 \%)$ cases were false negative. The sensitivity and specificity were $78.57 \%$ and $75 \%$ respectively. The positive and negative predictive values were $50 \%$ and $91.67 \%$ respectively with $75.9 \%$ accuracy. Statistically no significant difference was noted between H\&E and IHC stains ( $p=0.057)$.

With Giemsa (fig 2) $H$. pylori organisms were detected in $13(22.4 \%)$ cases. $4(6.9 \%)$ cases were found to be false positive and $5(8.6 \%)$ cases were false negative. The sensitivity and specificity were $64.28 \%$ and $90.9 \%$ respectively. The positive and negative predictive values were $69.23 \%$ and $88.8 \%$ respectively with $84.5 \%$ accuracy. However, statistically no significant difference was noted between Giemsa and IHC stains $(p=1.000)$.

Of the 58 cases studied, H\&E stained sections revealed mild colonization in 15 (25.9\%), moderate colonization in $4(6.9 \%)$ and severe colonization in $3(5.2 \%)$. No colonization was detected in the remaining $36(62.1 \%)$ cases. On correlating with IHC positivity it was seen that $\mathrm{H} \& \mathrm{E}$ detected all cases of severe colonization. However, there was disparity noted in the detection of mild and moderate cases of colonization. IHC confirmed the presence of the organism in $6(10.3 \%)$ cases of mild colonization and $2(3.45 \%)$ cases of moderate colonization, while H\&E detected $H$. pylori in 15 cases and 4 cases respectively. 
Table 1: Pattern of gastritis of the studies cases

\begin{tabular}{|l|l|}
\hline Lesion & No. $(\%)$ \\
\hline Inflammation & \\
\hline Absent & $3(5.2)$ \\
\hline Mild & $33(56.9)$ \\
\hline Moderate & $20(34.5)$ \\
\hline Severe & $2(3.4)$ \\
\hline Activity & $26(44.8)$ \\
\hline Glandular Atrophy & $7(12.1)$ \\
\hline Intestinal Metaplasia & $12(20.7)$ \\
\hline
\end{tabular}

Table 2: Comparison of Results of H\&E and Gold Standard IHC

\begin{tabular}{|l|l|l|l|l|}
\hline \multicolumn{2}{|c|}{} & \multicolumn{2}{|l|}{ IHC } & \multirow{2}{*}{ Total (\%) } \\
\cline { 3 - 5 } \multicolumn{2}{|c|}{} & $+(\%)$ & $-(\%)$ & \\
\hline \multirow{3}{*}{ H\&E } & $+(\%)$ & $11(19)$ & $11(19)$ & $\mathbf{2 2}(38)$ \\
\cline { 2 - 5 } & $-(\%)$ & $3(5.2)$ & $33(56.8)$ & $\mathbf{3 6}(62)$ \\
\hline \multicolumn{2}{|l|}{ Total (\%) } & $\mathbf{1 4}(24.1)$ & $\mathbf{4 4}(\mathbf{7 5 . 9})$ & $\mathbf{5 8}$ \\
\hline
\end{tabular}

Table 3: Comparison of Results of Giemsa and Gold Standard IHC

\begin{tabular}{|l|l|l|l|l|}
\hline \multicolumn{2}{|c|}{} & \multicolumn{2}{|l|}{ IHC } & \multirow{2}{*}{ Total (\%) } \\
\cline { 3 - 5 } \multicolumn{2}{|c|}{ Giemsa } & $+(\%)$ & $-(\%)$ & \\
\hline & $+(\%)$ & $9(15.5)$ & $4(6.9)$ & $\mathbf{1 3}(\mathbf{2 2 . 4 )}$ \\
\cline { 2 - 5 } & $-(\%)$ & $5(8.6)$ & $40(68.9)$ & $\mathbf{4 5}(\mathbf{7 7 . 5})$ \\
\hline \multicolumn{2}{|l|}{ Total (\%) } & $\mathbf{1 4}(\mathbf{2 4 . 1})$ & $\mathbf{4 4}(\mathbf{7 5 . 9})$ & $\mathbf{5 8}$ \\
\hline
\end{tabular}

Table 4: Validity of H\&E and Giemsa

\begin{tabular}{|l|l|l|l|l|}
\hline & Sensitivity & Specificity & PPV & NPV \\
\hline H\&E & $78.57 \%$ & $75 \%$ & $50 \%$ & $91.67 \%$ \\
\hline Giemsa & $64.28 \%$ & $90.9 \%$ & $69.23 \%$ & $88.8 \%$ \\
\hline
\end{tabular}

Table 5: Comparison of degree of colonization in H\&E and IHC

\begin{tabular}{|c|c|c|c|}
\hline \multirow[b]{2}{*}{ Bacterial Colonization } & \multirow[b]{2}{*}{ H\&E No. (\%) } & \multicolumn{2}{|l|}{ IHC } \\
\hline & & $\begin{array}{l}\text { H. pylori + No. } \\
(\%)\end{array}$ & $\begin{array}{l}\text { H. pylori -No. } \\
(\%)\end{array}$ \\
\hline Mild & $15(25.9)$ & $6(10.3)$ & $9(15.5)$ \\
\hline Moderate & $4(6.9)$ & $2(3.45)$ & $2(3.45)$ \\
\hline Marked & $3(5.2)$ & $3(5.2)$ & $0(0.0)$ \\
\hline Total & $22(37.9)$ & $11(18.95)$ & $11(18.95)$ \\
\hline
\end{tabular}

Table 6: Comparison of degree of inflammation with presence of $\mathrm{H}$. pylori

\begin{tabular}{|l|l|l|}
\hline \multirow{2}{*}{ Degree of Inflammation (n) } & H. pylori (IHC) & - No. $(\boldsymbol{\%})$ \\
\cline { 2 - 3 } & + No. $(\%)$ & $24(72.7)$ \\
\hline Mild (33) & $9(27.3)$ & $16(80)$ \\
\hline Moderate (20) & $4(20)$ & $1(50)$ \\
\hline Marked (2) & $1(50)$ & $\mathbf{4 1}(\mathbf{7 4 . 5})$ \\
\hline Total (55) & $\mathbf{1 4}(\mathbf{2 5 . 5})$ & \\
\hline
\end{tabular}


Table 7: Colonization of $\boldsymbol{H}$. pylori by parameters of gastritis

\begin{tabular}{|l|l|l|}
\hline \multirow{2}{*}{ Pattern of Gastritis (n) } & H. pylori & \multicolumn{2}{|l|}{ No. (\%) } \\
\cline { 2 - 3 } & + No. (\%) & $18(69.2)$ \\
\hline Activity (26) & $8(30.8)$ & $5(71.4)$ \\
\hline Atrophy (7) & $2(28.6)$ & $12(100.0)$ \\
\hline Metaplasia (12) & $0(0.0)$ & \\
\hline
\end{tabular}

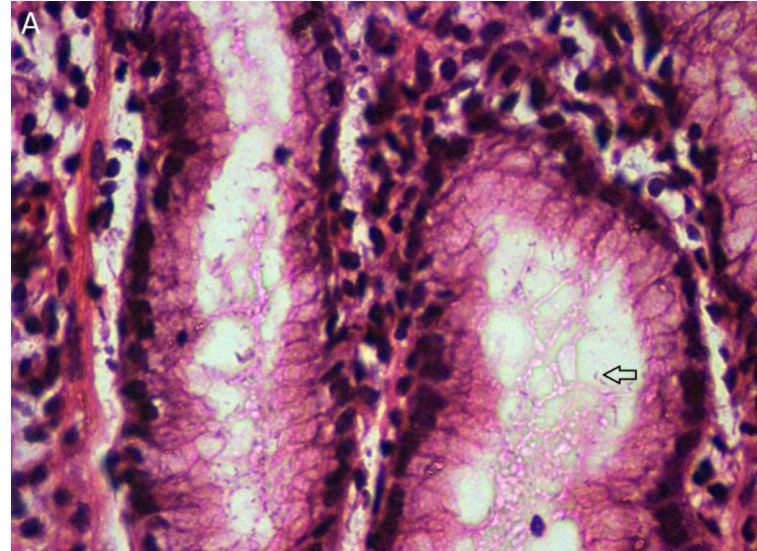

Fig 1: Microscopic appearance of $H$. pylori as seen with H\&E staining

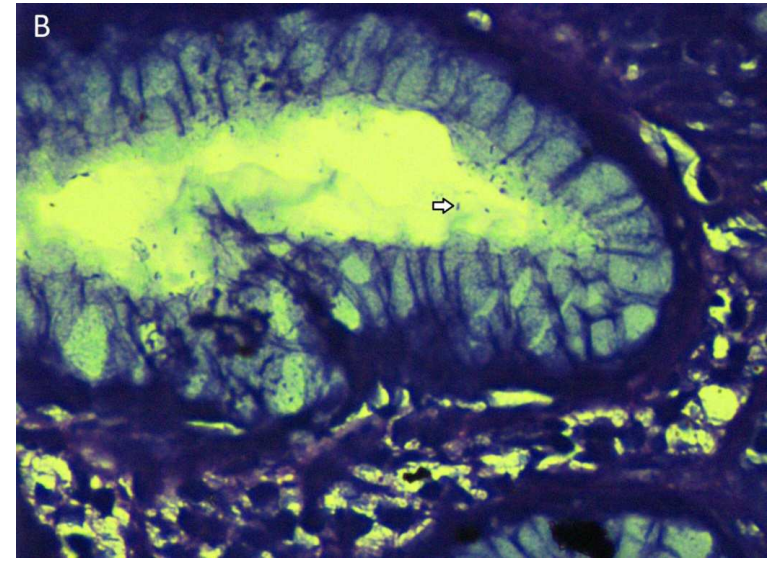

Fig 2: Microscopic appearance of $\mathrm{H}$. pylori as seen with Giemsa staining

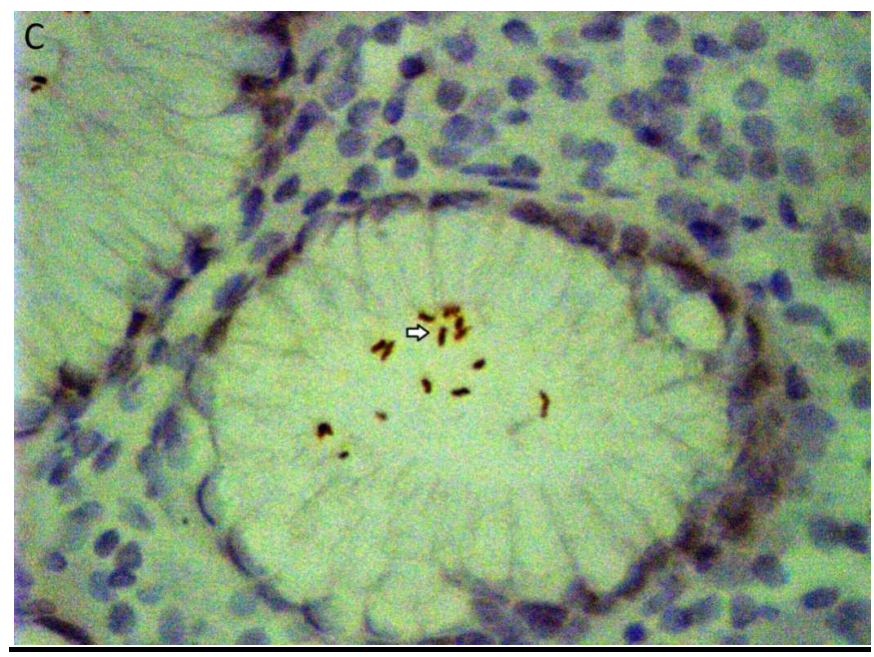

Fig 3: Microscopic appearance of $H$. pylori as seen with IHC staining

Most of the cases studied showed signs of inflammation. On correlating the grade of inflammation and $H$. pylori positivity with IHC the results obtained were such. The organism was found in $9(27.3 \%)$ cases of mild inflammation, 4 $(20 \%)$ cases of moderate inflammation and $1(50 \%)$ case of severe inflammation.

H. pylori colonization, with IHC, was also found in cases showing activity and glandular atrophy. It was seen in 8 (30.8\%) cases of activity, 2 (28.6\%) cases of glandular atrophy. The organism however, was not seen to be associated with intestinal metaplasia. On the other hand, of the 14 IHC positive cases, $8(57.1 \%)$ cases showed activity while no activity was noted in the remaining $6(42.9 \%)$ cases. The inter-observer variation kappa (k) was found to be 0.47 which suggests moderate agreement. 


\section{Discussion}

The study was started with the aim of comparing, the easily available and widely used staining methods $\mathrm{H} \& \mathrm{E}$ and Giemsa against the gold standard IHC among patients with clinical suspicion of $H$. pylori infection.

The study showed sensitivity of $78.57 \%$, specificity of $75 \%$ and a false positive rate of $19 \%$ for the routine H\&E staining method. The low sensitivity was also noted by Shukla et al [6] and Intisar et al [13] in their studies. It was suggested that the low sensitivity and high false positive rate was due to the lack of contrast between the organism and the surrounding gastric mucosa. [13] This could also be because of the confusion which arises due to gastric secretions or eosinophilic debris seen in $\mathrm{H} \& \mathrm{E}$ stained sections.

Giemsa staining showed low sensitivity (64.28\%) but a high specificity (90.9\%). Similar results were obtained in the study by Laine et al. [15]; while the study by $\mathrm{H}$. R. Wabinga [9] showed higher sensitivity (85\%). In our study, Giemsa stained sections revealed organisms in 13 (22.4\%) cases compared to the $22(38 \%)$ cases detected by $H \& E$. Giemsa had a lesser rate of false positives (6.9\%) than H\&E. In the present study Giemsa showed higher specificity than $\mathrm{H} \& \mathrm{E}$ which was also noted by other studies $[3,6,9]$. The low sensitivity, also noted by Tajalli et al. [3], makes it less reliable for detection of H. pylori infection. The low sensitivity could be due to the fact that the bacilli adherent to the glandular epithelium, especially in mild degree of colonization could be missed as both appear blue on Giemsa staining.

In this present study the association of $H$. pylori with inflammation (mild: $27.3 \%$, moderate: $20 \%$, severe: $50 \%)$ [Table 5] was not in accordance with the association seen in the study conducted in Africa (mild: 55\%, moderate: $63.2 \%$, severe: $42.9 \%$ ) [9]; or that conducted in Duhok, Iraq (mild: $14 \%$, moderate: 72.6\%, severe: $36.8 \%$ ). [13] Activity was seen to be associated with $H$. pylori positivity in $30.8 \%$ of cases. Also $57.1 \%$ of $H$. pylori positive cases showed evidence of activity. Such significant association between activity and presence of $H$. pylori infection was also noted in study by Toulaymat $\mathrm{M}$ et al. [5] Studies have suggested activity to be almost always associated with $H$. pylori infection. $[16,17]$ In our study the organism was seen to be associated most, with severe inflammation. Hence, the presence of severe form of inflammation and activity should prompt a careful scrutiny of the sections for the presence of the organisms.

H. pylori infection is an important risk factor for the development of gastric atrophy and intestinal metaplasia. [18] In this study [Table 7] glandular atrophy was noted in $28.6 \%$ of cases with $H$. pylori colonization. The association between these parameters has been shown in other studies. In the study conducted by Toulaymat et al [5] it was $69.4 \%$ and it was found to be $48.3 \%$ and in the study done by Intisar et al [13]. This variation may be explained by the finding of another study which showed a poor inter-observer variation value $(\mathrm{k}=0.31)$ for the evaluation of atrophy [19]. In contrast to the findings of Toulaymat et al [5] and Shukla et al [6] of finding intestinal metaplasia in H. pylori positive cases, no such association could be established by our study. It is known for the organisms to be absent in the region of metaplasia and must be looked for in other parts of the section.

The study also considered the inter-observer variation between two observers for H\&E stained sections which was found to be $\mathrm{k}=0.47$, suggesting moderate agreement. Study by Aydin et al [19] also suggested moderate agreement with $\mathrm{k}=0.56$. The organism is known to have a patchy distribution, especially in mild infection. Therefore this variation may be reduced by screening multiple sections for the organism.

\section{Conclusion}

Giemsa staining showed a lower sensitivity compared to H\&E. However, Giemsa staining showed a high specificity and lower false positive rate than H\&E. Therefore, Giemsa may be used complementary to $\mathrm{H} \& \mathrm{E}$. Use of IHC further reduces the rate of false positive results. There is a strong association between activity and the presence of organisms, especially with mild infections. Considering this and the high cost of IHC, IHC can be restricted to cases showing activity, cases without clear diagnosis with ancillary stains and those cases with a strong clinical suspicion.

\section{Acknowledgements:}

Grants from:

- Indian Council of Medical Research

- Medical Education Research Trust

- M S Ramaiah Medical College

Samples: Dr. Umesh Jalihal, Professor and HOD, Dept. of Gastroenterology, M S Ramaiah Hospitals. 
Statistical Analysis: Mr. Shivraj, Ms. Radhika, M S Ramaiah Medical College

Funding: Nil,Conflict of interest: None.

Permission of IRB: Yes

\section{References}

1. Yantiss RK, Lamps LW. To stain or not to stain...That remains the question. Am J Clin Pathol 2012 Mar; 137(3):343-5. doi: 10.1309/AJCPXC4K9IOVUEKT.

2. Kusters JG, van Vliet AH, Kuipers EJ. Pathogenesis of Helicobacter pylori infection. Clin Microbiol Rev. 2006 Jul; 19(3):449-90. doi:10.1128/CMR.00054-05

3. Tajalli R, Nobakht M, Mohammadi-Barzelighi H, Agah S, Rastegar-Lari A, Sadeghipour A. The immunohistochemistry and toluidine blue roles for Helicobacter pylori detection in patients with gastritis. Iran Biomed J. 2013 Jan; 17(1): 36-41. doi: 10.6091/IBJ.1094.2012.

4. Garza-González E, Perez-Perez GI, MaldonadoGarza HJ, Bosques-Padilla FJ. Diagnosis and treatment of $\mathrm{H}$. pylori infection. World J Gastroenterol $2014 \mathrm{Feb}$ 14; 20(6): 1438-1449. doi:10.3748/wjg.v20.i6.1438.

5. Toulaymat M, Marconi S, Garb J, Otis C, Nash S. Endoscopic Biopsy Pathology of Helicobacter pylori Gastritis Comparison of Bacterial Detection by Immunohistochemistry and Genta Stain. Arch Pathol Lab Med. 1999 Sep; 123(9):778-781.

6. Shukla S, Pujani M, Agarwal A, Pujani M, Rohtagi A. Correlation of Serology with Morphological Changes in Gastric Biopsy in Helicobacter Pylori Infection and Evaluation of Immunohistochemistry for H. Pylori Identification. Saudi J Gastroenterol. 2012 Nov-Dec; 18(6): 369-374. doi: 10.4103/13193767.103428

7. Tonkic A, Tonkic M, Lehours $\mathrm{P}$, Mégraud F. Epidemiology and diagnosis of Helicobacter pylori infection. Helicobacter 2012 Sep; 17(Suppl. 1): 1-8. doi: 10.1111/j.1523-5378.2012.00975.x.

8. Chey WD, Wong BC. American College of Gastroenterology Guideline on the Management of Helicobacter pylori Infection. Am J Gastroenterol.2007 Aug; 102(8):1808-25.
9. Wabinga HR. Comparison of immunohistochemical and modified Giemsa stains for demonstration of Helicobacter pylori infection in an African population. Afr Health Sci. 2002 Aug; 2(2):52-5.

10. Rotimi O, Cairns A, Gray S, Moayyedi P, Dixon M. Histological identification of Helicobacter pylori: comparison of staining methods. J Clin Pathol. 2000 Oct; 53(10):756-759. doi: 10.1136/jcp.53.10.756.

11. Hartman DJ, Owens SR. Are routine ancillary stains required to diagnose Helicobacter infection in Gastric biopsy specimens? An institutional quality assurance review. Am J. Clin Pathol. 2012 Feb; 137(2):255-260. doi: 10.1309/AJCPD8FFBJ5LSLTE.

12. Wang XI, Zhang S, Abreo F, Thomas J. The role of routine immunohistochemistry for Helicobacter pylori in gastric biopsy. Ann Diagn Pathol. 2010 Aug; 14(4):256-259. doi: 10.1016/j.anndiagpath.2010.05.002.

13. Intisar S, Pity Azad M, Baizeed. Identification of Helicobacter Pylori in Gastric Biopsies of Patients with Chronic Gastritis: Histopathological And Immunohistochemical Study. Duhok Med J 2011 Jun; 5(1): 69-77.

14. Dixon MF, Genta RM, Yardley JH, Correa P. Classification and Grading of Gastritis. The updated Sydney System. International Workshop on the Histopathology of Gastritis, Houston 1994. Am J Surg Pathol. 1996 Oct; 20(10):1161-81.

15. Laine L, Lewin DN, Naritoku W, Cohen H. Prospective comparison of H\&E, Giemsa, and Genta stains for the diagnosis of Helicobacter pylori. Gastrointest Endosc. 1997 Jun; 45 (6): 463-7.

16. Stolte M, Stadelmann O, Bethke B, Burkard G. Relationships between the degree of Helicobacter pylori colonization and the degree of activity of gastritis, surface epithelial degeneration and mucus secretion. Z Gastroenterol. 1995 Feb; 33(2):89-93.

17. Miehlke S, Hackelsberger A, Meining A, Hatz R, Lehn N, Malfertheiner P, Stolte M, Bayerdörffer E. Severe expression of corpus gastritis is characteristic in gastric cancer patients infected with Helicobacter pylori. Br J Cancer. 1998 Jul; 78(2):263-266. 
18. Zhang C, Yamada N, Wu YL, Wen M, Matsuhisa T, Matsukura N. Helicobacter pylori infection, glandular atrophy and intestinal metaplasia in superficial gastritis, gastric erosion, erosive gastritis, gastric ulcer and early gastric cancer. World J Gastroenterol. 2005 Feb 14; 11(6):791-6. doi: 10.3748/wjg.v11.i6.791.
19. Aydin O, Egilmez R, Karabacak T, Kanik A. Interobserver variation in histopathological assessment of Helicobacter pylori gastritis. World J Gastroenterol $2003 \quad$ Oct $15 ; \quad 9(10): 2232-2235$. doi: 10.3748/wjg.v9.i10.2232.

\section{How to cite this article?}

Pranjal Sharma R, Kalpana Kumari M.K. Histological identification of Helicobacter pylori: comparison of staining methods. Int J Med Res Rev 2015;3(10):1167-1173. doi: 10.17511/ijmrr.2015.i10.211. 